\title{
High expression of RBM8A predicts poor patient prognosis and promotes tumor progression in hepatocellular carcinoma
}

\author{
RONG LIANG $^{1}$, YAN LIN ${ }^{1}$, JIA-ZHOU YE ${ }^{2}$, XUE-XIN YAN ${ }^{1}$, ZHI-HUI LIU ${ }^{1}$, \\ YONG-QIANG LI ${ }^{1}$, XIAO-LING LUO ${ }^{1}$ and HAI-HONG YE $^{3}$ \\ ${ }^{1}$ First Department of Chemotherapy and ${ }^{2}$ Department of Hepatobilliary Surgery, Affiliated Tumor Hospital \\ of Guangxi Medical University, Nanning, Guangxi 530022; ${ }^{3}$ Department of Hepatobilliary Surgery, \\ Affiliated Minzu Hospital of Guangxi Medical University, Nanning, Guangxi 530001, P.R. China
}

Received September 5, 2016; Accepted January 30, 2017

DOI: $10.3892 / o r .2017 .5457$

\begin{abstract}
Hepatocellular carcinoma (HCC) is a huge threat for human health worldwide. As a complicated tumor, the molecular basis for HCC development especially metastasis requires exploration. Although RNA binding motif (RBM) proteins are closely related to various cancers, the clinical importance and underlying mechanisms of RBM8A in $\mathrm{HCC}$ remain elusive. In this study, we found that RBM8A was highly expressed in $\mathrm{HCC}$ tumor tissues compared to normal liver tissues. Overexpression of RBM8A was associated with HbsAg and Edmondson pathological grading. Moreover, Kaplan-Meier survival analysis showed that high expression of RBM8A was related to the poor overall survival and progression-free survival of patients with HCC. Gain- and loss-of-function experiments further demonstrated that RBM8A promoted tumor cell migration and invasion in $\mathrm{HCC}$ via activation of epithelial-mesenchymal transition signaling pathway. It is also noteworthy that RBM8A is required for tumor cell proliferation and anti-apoptosis in HCC. Altogether, our results revealed a close relationship between RBM8A and HCC prognosis as well as a critical tumor-promoting function of RBM8A in $\mathrm{HCC}$ progression, suggesting that RBM8A might be a potential bio-marker and drug target in HCC therapy.
\end{abstract}

\section{Introduction}

Hepatocellular carcinoma (HCC) is a primary malignancy of the liver with over 500,000 people affected. HCC is now the third leading cause of cancer deaths worldwide which attracts intensive research (1). The occurrence and development of $\mathrm{HCC}$ is a complex process involving multi-genes, multi-steps

Correspondence to: Professor Hai-Hong Ye, Department of Hepatobilliary Surgery, Affiliated Minzu Hospital of Guangxi Medical University, 232 Mingxiu East Road, Nanning, Guangxi 530001, P.R. China

E-mail: article811@foxmail.com

Key words: RBM8A, hepatocellular carcinoma, prognosis, epithelial-mesenchymal transition, invasion and multi-stages. The invasion and metastasis of HCC is closely linked with HCC patient's treatment and prognosis (2). Thus, it is of great significance to reveal HCC specific genes and investigate the mechanism of aberrant molecules in the occurrence and development of HCC.

The recently identified RNA-binding motif protein $8 \mathrm{~A}$ (RBM8A, also known as Y14) belongs to the RNA-binding motif protein (RBM) family (3). Scientists have focused on this protein family for its diverse functions. These proteins bind to RNA and tightly control gene expression, cell cycle, apoptosis and RNA splicing (4-8). RBM8A is a vital apoptosis regulator and loss of RBM8A leads to increased apoptosis. Ishigaki et al (9) found that loss of RBM8A inhibited the proliferation of HeLa and A549 cells and cells were blocked at M phase. Importantly, they found the abnormal centrosome in cancer cells, which is a symbolic feature of apoptosis, indicating the loss of RBM8A directly regulate cell apoptosis. Moreover, downregulated RBM8A led to decreases of pro-apoptotic genes such as Bcl-Xs belonging to Bcl-2 family, Bim and Mcl1S $(10,11)$. These studies showed that PBM8A participated in various steps of apoptosis. Evidence also demonstrated that RBM8A control tumor occurrence and development via regulating mRNA splicing.

The function of RBM8A in HCC is poorly understood. Although Zindy et al (12) found that RBM8A was upregulated in liver cancer as a target gene of re-establishment of extracellular matrix, metabolism and post-transcription regulation, the function of RBM8A in HCC remain largely unknown. Hence, we analyzed the expression level of RBM8A in patient HCC tumor samples and evaluated the relationship of RBM8A expression level and clinicopathological factors and prognosis. We further explored the function of RBM8A in HCC tumor cell migration, invasion as well as apoptosis.

\section{Materials and methods}

Patient data. A total of 105 surgical tissue samples of HCC were collected from patients at Affiliated Tumor Hospital of Guangxi Medical University after obtaining the subject informed consent and institutional review board approval.

Cell lines. The human HCC cell lines were purchased from Stem Cell Bank, Chinese Academy of Sciences. Bel-7404, 
and HL-7702 cells were maintained in Dulbecco's modified Eagle's medium (DMEM). MHCC97H, a high-metastatic human HCC, was established as reference (13). The media were supplemented with $10 \%$ heat-inactivated fetal bovine serum (FBS; Invitrogen, Carlsbad, CA, USA), penicillin (100 U/ml), and streptomycin $(100 \mu \mathrm{g} / \mathrm{ml})$ in a humidified atmosphere of $5 \% \mathrm{CO}_{2}$ at $37^{\circ} \mathrm{C}$. All the cells were confirmed to be free from mycoplasma contamination.

Establishment of stable cell lines in which RBM8A was overexpressed or knocked down. The siRNA sequences were: sh1: 5'-AGAGCATTCACAAACTGAA-3'; sh2: 5'-CATCAGCG TTGACTGGTGT-3'; sh3: 5'-GCAACAGGTCTAGGGT TAAGG-3'. Lentiviral vectors encoding shRNA were designed based on the sequences of siRNA to knock down RBM8A expression (RBM8A-KD). These vectors were constructed by Hanyin Co. (Shanghai, China). The recombinant lentiviruses (KD) and the negative control (NC) lentivirus (Hanyin Co.) were prepared and titered to 109 transfection units (TU)/ml. To obtain stable cell lines, cells were seeded in 6-well plates and infected with virus and polybrene the following day. Positive clones were selected with puromycin for 14 days to establish the stable cell lines. Additionally, the lentiviruses expressing the RBM8A sequence (OE) and the negative control lentivirus (Vector) were constructed by Hanyin Co. RBM8A-OE and control stable cell lines were then established. The efficiency of RBM8A knockdown and overexpression was confirmed by qRT-PCR.

Western blot analysis. Protein lysates (50 $\mu \mathrm{g}$ per lane) were separated by sodium dodecyl sulfate (SDS)-polyacrylamide gel electrophoresis and transferred onto nitrocellulose membranes. After blocking with $5 \%$ fat-free milk, the membranes were incubated with primary antibodies (1:500 dilution) at $4{ }^{\circ} \mathrm{C}$ overnight, followed by horseradish peroxidase-conjugated secondary antibodies (1:3,000 dilution). Anti-human RBM8A (Santa Cruz Biotechnology, sc-32312), anti-human actin (Proteintech, HRP-60008), anti-human Snail (Cell Signaling Technology, 3879), anti-human p-STAT3 (Cell Signaling Technology, 9131), anti-human STAT3 (Cell Signaling Technology, 12640), anti-human fibronectin (Sigma-Aldrich, F3648), anti-human vimentin (Cell Signaling Technology, 5741), anti-human Notch (Abaways, CY52444) and donkey anti-Rabbit IgG (Cell Signaling Technology, 7074) were used in this study. Immune-reactive proteins were visualized by enhanced chemiluminescence (ECL).

Histology and immunohistochemistry. Histological and immunohistochemical analyses were performed using an HRP kit (UltraTek; Scytek). Anti-human RBM8A antibody diluted 1:50 in blocking buffer were used as primary antibodies. The diaminobenzidine-stained specimens were visualized using a general optical microscope with a camera (AxioCam ICc5; Carl Zeiss). Images were processed with equivalent parameters using ZEN Light Edition software (Carl Zeiss).

Total RNA isolation and quantitative real-time PCR (qRT-PCR) analysis. Total RNA was isolated from liver cancer cells and tumor tissues using TRIzol reagent according to the manufacturer's directions (Invitrogen). cDNA was reverse tran-
Table I. Quantification of RBM8A protein levels in HCC tissues, adjacent tissues and normal non-carcinoma tissues.

\begin{tabular}{lccc}
\hline & & \multicolumn{2}{c}{ RBM8A/ $\beta$-actin } \\
\cline { 3 - 4 } Tissues & Cases & Mean \pm SD & P-value \\
\hline Carcinoma & 105 & $1.86 \pm 0.36$ & \\
Adjacent & 105 & $1.35 \pm 0.32$ & $0.000^{\mathrm{a}}$ \\
Non-carcinoma & 67 & $0.95 \pm 0.35$ & $0.000^{\mathrm{b}}$
\end{tabular}

${ }^{\mathrm{a}}$ Compared with carcinoma tissue. ${ }^{\mathrm{b} C}$ Compared with carcinoma tissue.

scripted from $1 \mathrm{mg}$ total RNA. qRT-PCR was performed with the SYBR Premix Ex Taq (Takara, Dalian, China). PCR primers were as follows: F: 5'-GCGTGAGGATTATGACAGCGTG-3', R: 5'-TTCGGTGGCTTCCTCATGGACT-3'.

The cycling conditions were as initial denaturation at $95^{\circ} \mathrm{C}$ for $5 \mathrm{~min}$, and then 36 cycles of denaturation at $95^{\circ} \mathrm{C}$ for $10 \mathrm{sec}$ and annealing at $60^{\circ} \mathrm{C}$ for $30 \mathrm{sec}$. The relative mRNA expression was calculated using the comparative $\mathrm{Ct}(\Delta \Delta \mathrm{Ct})$ method.

Wound-healing assay. The in vitro migration ability of $\mathrm{HCC}$ cells was assessed by wound-healing assay. Cells were plate in 6-well plates and the monolayer was artificially scratched with $10-\mu 1$ pipette tips. The wound areas were photographed 0 and $20 \mathrm{~h}$ after scratching and measured using a caliper. The wound-closure percentages were calculated using the following formula: [1-(current wound size/initial wound size)] x100.

Cell invasion assay. Cells were detached and re-suspended in a serum-free medium and seeded on the upper chamber of Matrigel-coated Transwell inserts with a pore size of $8 \mu \mathrm{m}$. The culture medium containing $10 \%$ FBS as a chemoattractant was added to the lower chamber. After $24 \mathrm{~h}$ incubation, the cells on the upper surface of the insert were gently removed with a cotton swab. Invading cells (lower surface of the insert) were fixed with 4\% paraformaldehyde (Sigma-Aldrich), stained with crystal violet, and counted under a microscope. Five random microscopic fields were examined for each insert.

Cell Counting Kit-8 (CCK-8) assay. The Bel7404 and BL7702 cells were seeded into 96 -well culture plates $\left(5 \times 10^{3}\right.$ cells/well). At 24, 48, 72 and $96 \mathrm{~h}, 10 \mu \mathrm{l}$ CCK-8 reagent (Beyotime Biotechnology) was added to each well and incubated for $4 \mathrm{~h}$. Then the absorbance values were read at a wavelength of $450 \mathrm{~nm}$ using a microplate reader (SpectraMax 250; GE Healthcare Life Sciences, Pittsburgh, PA, USA).

Flow cytometry analysis. Cells were seeded into 6-well plates at a density of $1 \times 10^{6}$ cells/well for $24 \mathrm{~h}$. Subsequently, the cells were collected and stained with the ANXA5 (Annexin V)-PE apoptosis detection kit (4A Biotech Co. Ltd., FXP018-100) according to the manufacturer's instructions and analyzed by flow cytometry (FACS Calibur, BD Biosciences, San Jose, CA, USA).

Statistical analyses. Unless stated otherwise, data are presented as mean \pm SD in the figures. A Student t-test was 
A

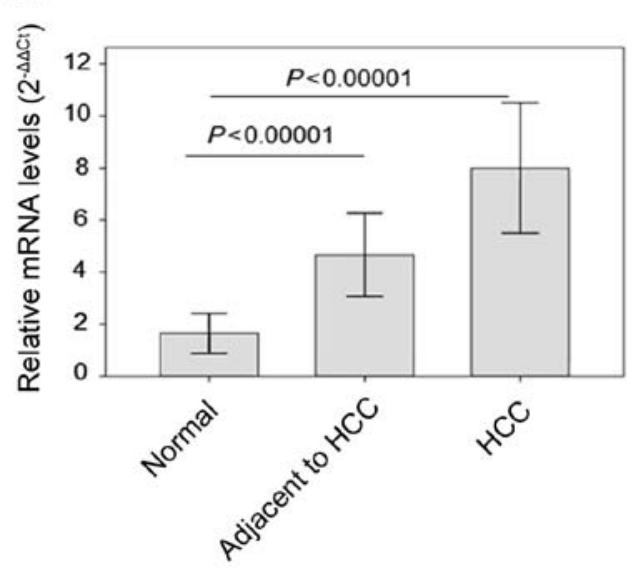

B

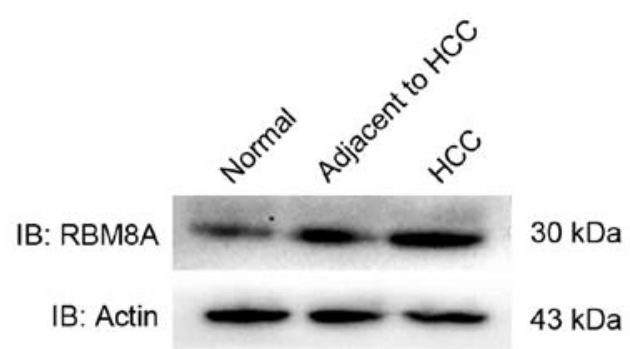

C

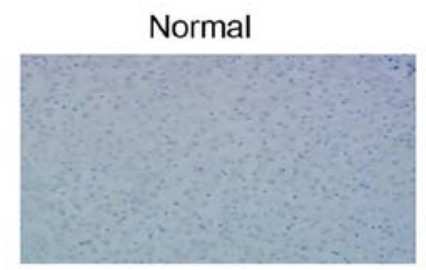

Adjacent to HCC

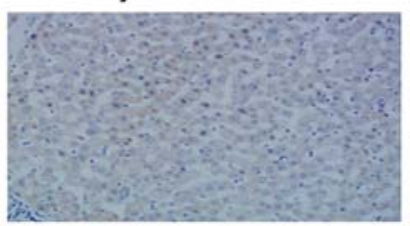

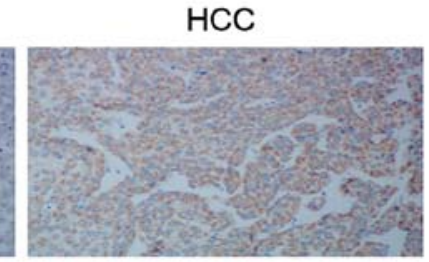

Figure 1. Expression of RBM8A in human normal liver tissue, carcinoma adjacent tissue and hepatocellular carcinoma. (A) mRNA expression level of RBM8A in human normal liver tissue, carcinoma adjacent tissue and hepatocellular carcinoma. (B) Protein expression level of RBM8A in human normal liver tissue, carcinoma adjacent tissue and hepatocellular carcinoma. (C) Immunohistochemistry staining of RBM8A in normal liver tissue, carcinoma adjacent tissue and hepatocellular carcinoma.

Table II. IHC results of RBM8A in HCC tissues, adjacent tissues and normal non-carcinoma tissues.

\begin{tabular}{lcccccr}
\hline Tissues & Cases & $\begin{array}{c}\text { Strong } \\
\text { staining }\end{array}$ & $\begin{array}{c}\text { Medium } \\
\text { staining }\end{array}$ & $\begin{array}{c}\text { Weak } \\
\text { staining }\end{array}$ & Negative & $\begin{array}{c}\text { Positive } \\
\text { rate }(\%)\end{array}$ \\
\hline Carcinoma & 105 & 18 & 46 & 28 & 13 & 87.62 \\
Adjacent & 105 & 3 & 28 & 33 & 41 & 60.95 \\
Non-carcinoma & 67 & 0 & 1 & 2 & 64 & 4.47 \\
\hline
\end{tabular}

${ }^{\mathrm{a} C}$ Compared with carcinoma tissue. ${ }^{\mathrm{b}}$ Compared with carcinoma tissue.

performed to compare the in vitro data. The Kaplan-Meier survival analysis was generated using SPSS version 13.0 (SPSS Inc., Chicago, IL, USA). One-way analysis of variance was initially performed to determine whether overall statistically significant changes occurred before using two-tailed paired or unpaired Student's t-tests. Multiple test-adjusted P-values of $<0.05$ were considered statistically significant. All statistical tests were two-sided.

\section{Results}

RBM8A is upregulated in hepatocellular carcinoma. In order to explore the function of RBM8A in hepatocellular carcinoma (HCC), we first detected the expression of RBM8A in normal liver tissues, carcinoma adjacent tissues and HCC tissues. Of note, we found that the mRNA level of RBM8A was significantly higher in HCC $(7.99 \pm 5.49)$ compared to carcinoma adjacent tissues $(4.66 \pm 3.78)$ as well as normal liver tissues $(1.64 \pm 1.53)$. We also noted that the mRNA level of RBM8A in carcinoma adjacent tissue was significantly higher than normal liver tissues (Fig. 1A). The western blotting results showed that RBM8A protein was detectable in all tissues and the protein level of RBM8A was consistently higher in HCC tissues $(1.86 \pm 0.36)$ compared to carcinoma adjacent tissues $(1.35 \pm 0.32)$ as well as normal liver tissues $(0.95 \pm 0.35)$ demonstrating that RBM8A was upregulated in HCC (Fig. 1B and Table I).

To further validate the expression differences, we performed immunohistochemistry (IHC) against RBM8A on normal liver tissue, carcinoma adjacent tissue and HCC tissue. As shown in Fig. 1C, strong positive signals were observed in HCC samples with a positive rate of $87.6 \%$. Statistical analysis results indicated that RBM8A IHC signals were significantly higher in carcinoma tissues and signals in carcinoma adjacent tissue were also higher than normal liver tissue (Table II).

Relationships between RBM8A expression levels with clinical indexes and survival. To further investigate the relationship 
Table III. The strength of RBM8A expression in HCC tissues and the relationship of clinical index.

\begin{tabular}{|c|c|c|c|c|c|c|c|}
\hline \multirow[b]{2}{*}{ Characteristics } & \multirow[b]{2}{*}{ Cases } & \multicolumn{2}{|c|}{ RBM8A mRNA } & \multirow[b]{2}{*}{ P-value } & \multicolumn{2}{|c|}{ RBM8A protein } & \multirow[b]{2}{*}{ P-value } \\
\hline & & High & Low & & Positive & Negative & \\
\hline Gender & & & & 0.104 & & & 0.144 \\
\hline Male & 78 & 66 & 12 & & 71 & 7 & \\
\hline Female & 27 & 19 & 8 & & 21 & 6 & \\
\hline Age & & & & 0.079 & & & 0.463 \\
\hline$\geq 60$ & 35 & 25 & 10 & & 29 & 6 & \\
\hline$<60$ & 70 & 60 & 10 & & 63 & 7 & \\
\hline $\operatorname{AFP}(\mu \mathrm{g} / \mathrm{l})$ & & & & 0.516 & & & 0.433 \\
\hline$\geq 400$ & 78 & 62 & 16 & & 70 & 8 & \\
\hline$<400$ & 27 & 23 & 4 & & 22 & 5 & \\
\hline $\mathrm{HBsAg}$ & & & & 0.000 & & & 0.000 \\
\hline Positive & 82 & 73 & 9 & & 81 & 1 & \\
\hline Negative & 23 & 12 & 11 & & 11 & 12 & \\
\hline Diameter of tumor (CM) & & & & 0.248 & & & 0.013 \\
\hline$\geq 10$ & 28 & 20 & 8 & & 27 & 1 & \\
\hline $5-10$ & 50 & 41 & 9 & & 39 & 11 & \\
\hline$<5$ & 27 & 24 & 3 & & 26 & 1 & \\
\hline Hepatocirrhosis & & & & 0.329 & & & 0.58 \\
\hline Yes & 58 & 45 & 13 & & 54 & 4 & \\
\hline No & 47 & 40 & 7 & & 38 & 9 & \\
\hline Portal vein thrombosis & & & & 0.128 & & & 0.276 \\
\hline Yes & 42 & 31 & 11 & & 35 & 7 & \\
\hline No & 63 & 54 & 9 & & 57 & 6 & \\
\hline Child-Pugh score & & & & 0.283 & & & 0.670 \\
\hline A & 43 & 34 & 9 & & 37 & 6 & \\
\hline $\mathrm{B}$ & 58 & 49 & 9 & & 52 & 6 & \\
\hline $\mathrm{C}$ & 4 & 2 & 2 & & 3 & 1 & \\
\hline Edmondsom grades & & & & 0.004 & & & 0.003 \\
\hline $\mathrm{I}$ & 20 & 10 & 10 & & 12 & 8 & \\
\hline II & 45 & 40 & 5 & & 42 & 3 & \\
\hline III & 38 & 33 & 5 & & 36 & 2 & \\
\hline IV & 2 & 2 & 0 & & 2 & 0 & \\
\hline TNM & & & & 0.153 & & & 0.000 \\
\hline I & 24 & 16 & 8 & & 15 & 9 & \\
\hline II & 60 & 51 & 9 & & 58 & 2 & \\
\hline III & 21 & 18 & 3 & & 19 & 2 & \\
\hline IV & 0 & & & & & & \\
\hline
\end{tabular}

between RBM8A expression levels with clinical indexes at both mRNA and protein levels, we set the relative mRNA expression level 7.99 as a limit and divided the 105 cases into RBM8A mRNA high group and RBM8A mRNA low group. There were 85 cases in the RBM8A mRNA high group with IHC positive while 20 cases in the RBM8A mRNA low group. Noteworthy, our results revealed significant differences of RBM8A protein levels with different HBsAg levels, tumor diameters, TNM staging and Edmondson pathological grading.
We observed significant correlations between RBM8A high group with HBsAg, Edmondson pathological grading and TMN stages (Table III). However, no significant differences between RBM8A mRNA levels with tumor diameter and TNM staging. Thus, our result showed that at both $\mathrm{mRNA}$ and protein level, high level of RBM8A were associated with HBsAg and Edmondson pathological grading.

Non-parametric rank correlation analysis between RBM8A protein expression and Edmondson pathological grading 
A

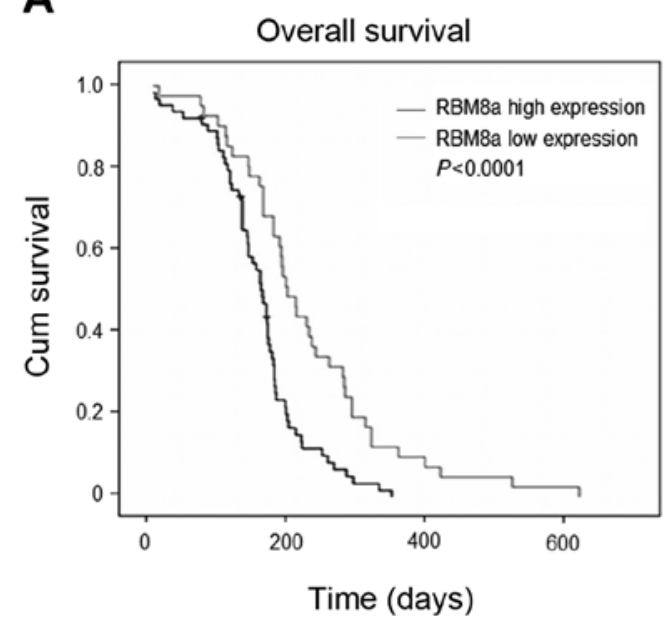

B

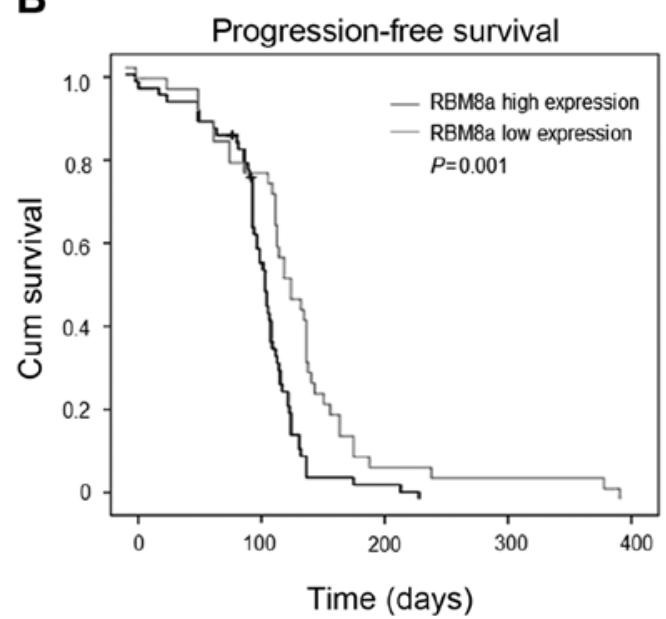

Figure 2. Relationship between RBM8A protein expression level and survival. (A) Relationship between RBM8A protein expression level and overall survival. (B) Relationship between RBM8A protein expression level and progression-free survival.

Table IV. Correlation between RBM8A protein expression level and Edmondson pathological grades.

\begin{tabular}{lccc}
$\begin{array}{l}\text { Edmondson } \\
\text { grades }\end{array}$ & No. & RBM8A/ $\beta$-actin & P-value \\
\hline I & 20 & $1.41 \pm 0.51$ & \\
II & 45 & $1.63 \pm 0.89$ & $0.004^{\mathrm{a}}$ \\
III & 38 & $2.21 \pm 0.63$ & $0.001^{\mathrm{b}}$ \\
IV & 2 & $2.50 \pm 0.71$ & $0.031^{\mathrm{c}}$ \\
\hline
\end{tabular}

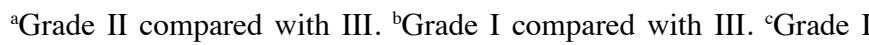
compared with IV.

showed that the Spearman correlation coefficient was 0.992 $(\mathrm{P}<0.001)$ indicating that RBM8A protein expression levels are closely associated with Edmondson pathological grading and stronger RBM8A protein level indicates lower degree of differentiation (Table IV).

Among the 105 cases, the follow-up periods varies from 3 days to 620 days without loss. All cases were fitted into the analysis and the follow-up rate was $100 \%$. We divide all cases into RBM8A protein high group (IHC staining: positive and strong positive, $n=64$ ) and RBM8A protein low group (IHC: staining weak positive and negative, $n=41$ ). By applying Kaplan-Meier survival curve, we calculated overall survival and progression-free survival and tested the statistic difference with Log-rank test. Our results showed that median of overall survival for RBM8A high expression group and RBM8A low expression group were 160 and 229 days, respectively, while median of progression-free survival for RBM8A high expression group and RBM8A low expression group were 90 and 114 days, respectively (Fig. 2). Both overall survival and progression-free survival in RBM8A low expression group were significantly longer than that in RBM8A high expression group indicating that patients with higher RBM8A expression are expecting shorter survival time.
Knockdown of RBM8A reduces cell migration and invasion. We next validated the results in human normal hepatic immortalized cell line and HCC cell lines. As expected, we found that RBM8A expressions were abundant in HCC cell lines Bel-7404 and MHCC-97H (Fig. 3A). On the contrary, RBM8A exhibited a low protein level in human hepatic immortalized cell line HL-7702. To further elucidate the function of RBM8A, we generated a set of shRNAs to knock down RBM8A in Bel-7404 cell line. Our results revealed that one of the shRNAs targeting 5'-AGAGCATTCACAAACTGAA-3' was able to reduce the mRNA of RBM8A by more than $80 \%$ (Fig. 3B).

Additionally, the protein level of RBM8A was also effectively downregulated with this shRNA while RBM8A was successfully rescued in Bel-7404 (Fig. 3C). To investigate the effect of RBM8A on cell migration and invasion, control cells, RBM8A knockdown cells and RBM8A rescued Bel-7404 cells were analyzed by migration assay. Our results showed that the migration of HCC cells were significantly reduced upon RBM8A knockdown while the RBM8A rescued group regained migration ability (Fig. 3D and E). We next applied gain of function experiment by overexpressing RBM8A in HL-7702 cell line. Both mRNA and protein level of RBM8A were significantly increased in HL-7702 RBM8A-OE cells (Fig. 4A and B). Moreover, the invasion ability of HCCs positively associated with the expression levels of RBM8A. (Fig. 4C and D). Thus, our results demonstrated that RBM8A promotes cell migration and invasion in HCC cells.

RBM8A regulates $H C C$ cell epithelial-mesenchymal transition. In order to elucidate the underlying mechanisms of RBM8A-mediated HCC migration and invasion, we investigated the epithelial-mesenchymal transition (EMT) markers and related signaling. We found a significant reduction of Snail and phosphorylated STAT3 in RBM8A knockdown Bel-7404 while unphosphorylated STAT3 remained stable indicating that EMT was impaired upon RBM8A knockdown (Fig. 5A). We noted a clear reduction of EMT markers fibronectin and vimentin in RBM8A knockdown Bel-7404 cells which also suggested a reduction of EMT (Fig. 5A). On the contrary, 
A
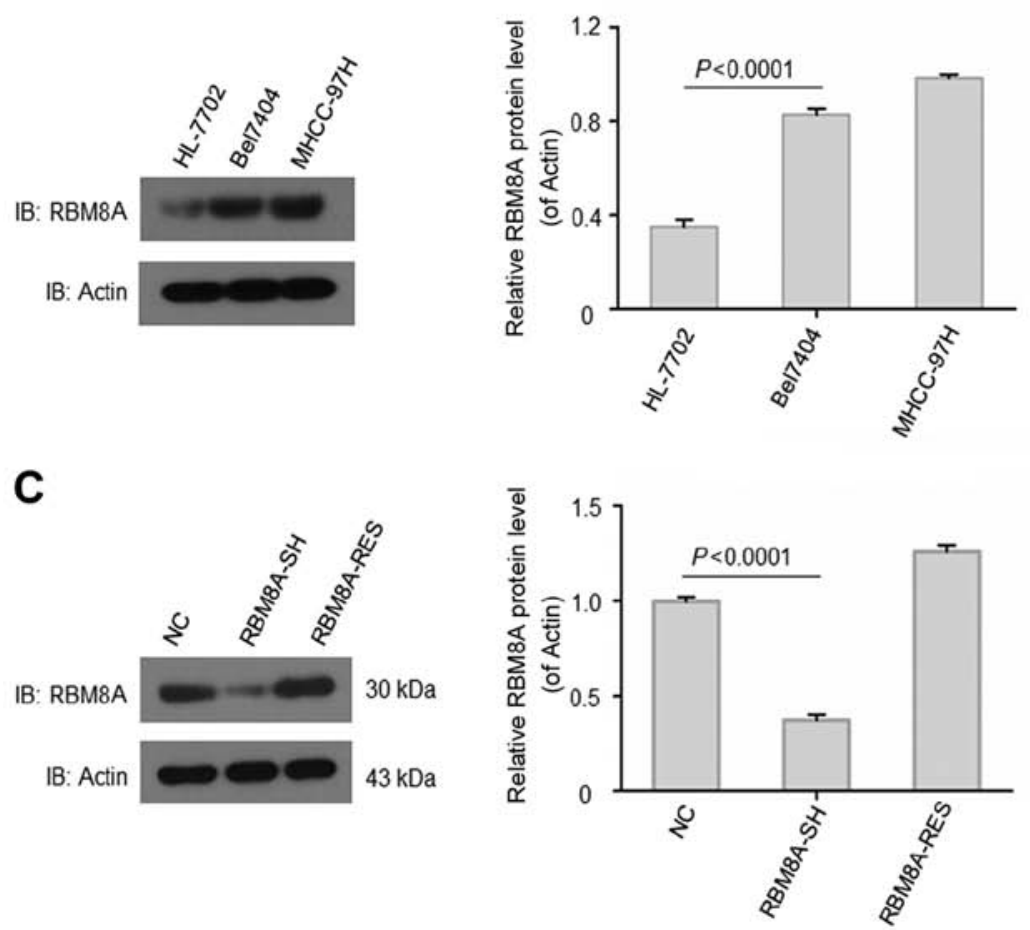

D
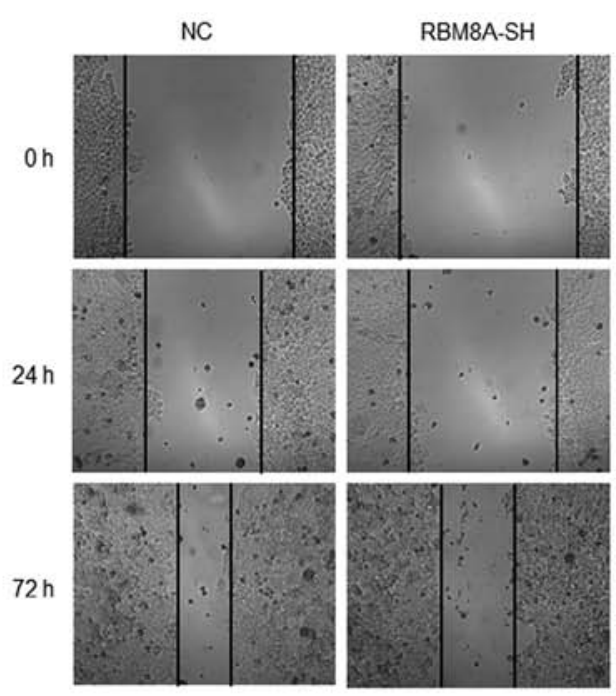
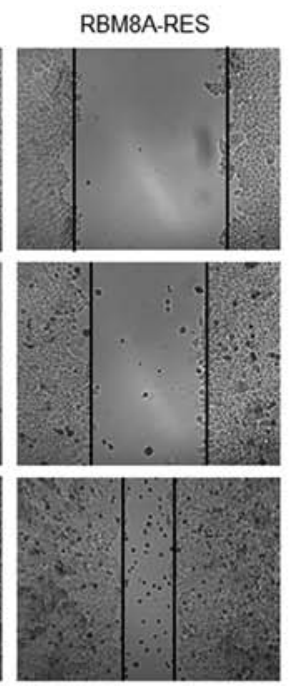

B

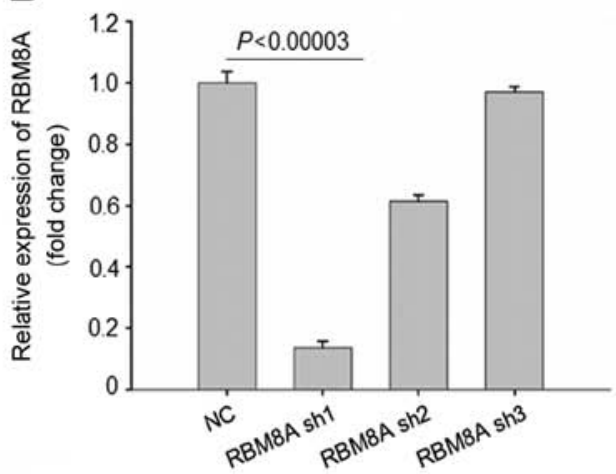

E

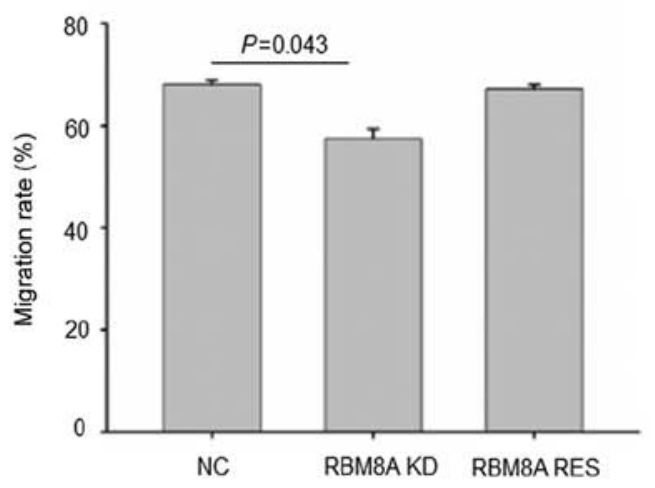

Figure 3. The role of RMB8A in migration. (A) Western blot analysis of RBM8A in different HCC cell lines. Quantitative data of western blot results were analyzed. (B) Knockdown of RBM8A with shRNAs. (C) RBM8A knockdown and rescue efficiency. Quantitative data of western blot results were analyzed. (D) Migration efficiency of control, RBM8A-SH and RBm8A-RES cell lines. (E) Quantification of migration efficiency in (D).

in RBM8A overexpressed HL-7702 cells, we observed an increase of Snail and phosphorylated STAT3 as well as fibronectin and vimentin (Fig. 5B) compared with control cells. Furthermore, immunofluorescence staining validated our western blotting results (Fig. 5C). All above results support that RBM8A is responsible for the epithelial-mesenchymal transition of HCC cells.

RBM8A has an important function in HCC cell survival. To further explore the role RBM8A plays in HCC cell, we examined whether RBM8A regulate cell proliferation in HCC cells. Noteworthy, our results displayed that knockdown of RBM8A in Bel-7404 cells significantly reduced the population of HCC after three days of cultivation while, ectopic expression of RBM8A in HL-7202 cells significantly increased the HCC population (Fig. 6A and B). Since RBM8A is a vital apoptosis regulator and loss of RBM8A leads to increased apoptosis (16), we examined the HCC apoptosis upon RBM8A knockdown or RBM8A overexpression. Accordingly, our FACS results showed a marked increase of apoptotic cells when RBM8A was knocked down in Bel-7404 (Fig. 6C). However, the number of apoptotic cells was greatly reduced in ectopic expression of RBM8A in HL-7202 cell line (Fig. 6D).

Taken together, our results demonstrated that high expression of RBM8A can predict poor patient prognosis and promote tumor progression in HCC cells. 
A

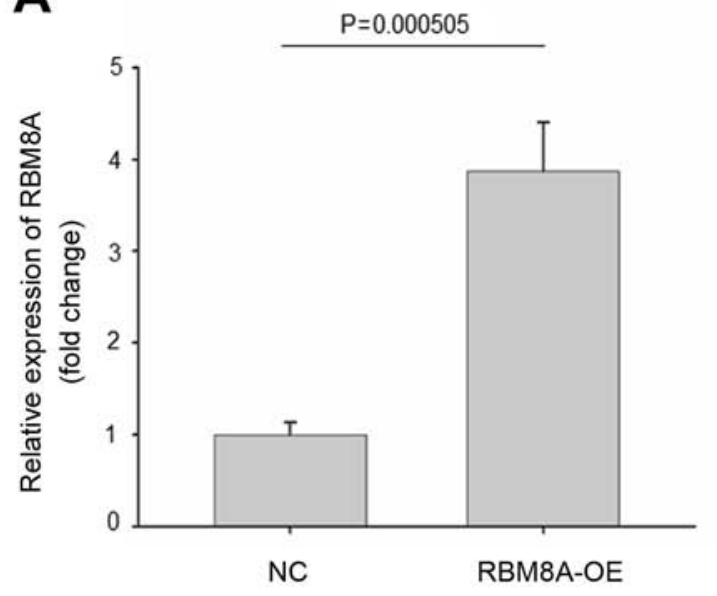

C
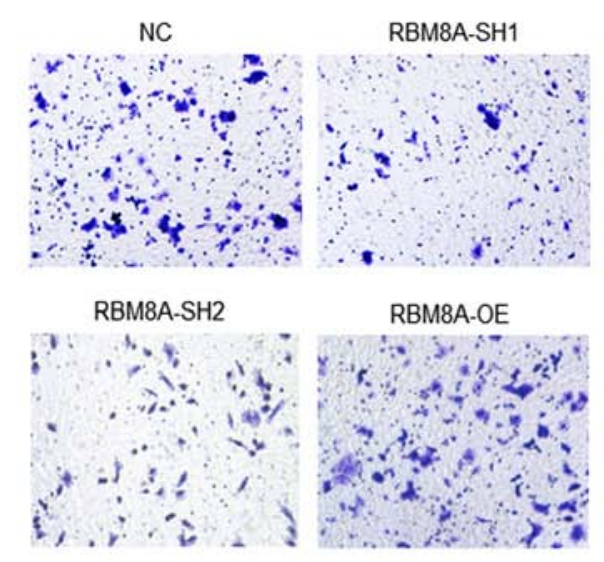

B

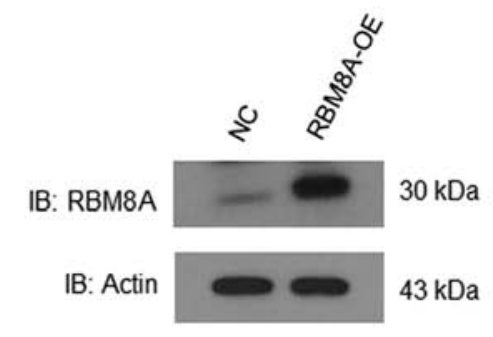

D

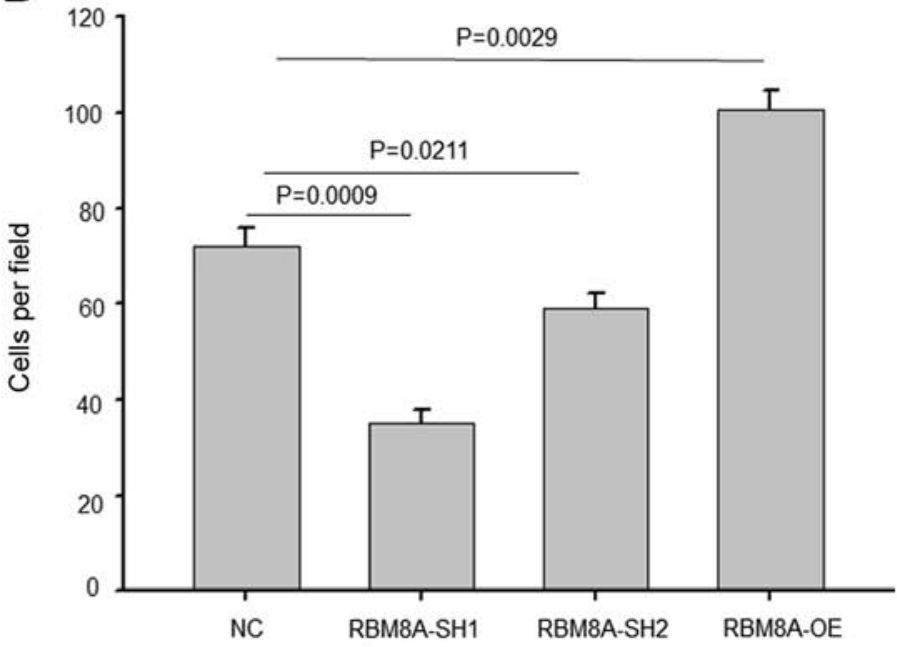

Figure 4. The role of RMB8A in invasion. (A) Overexpression efficiency of RMB8A at mRNA level. (B) Overexpression efficiency of RMB8A at protein level. (C) Invasion efficiency of control, RBM8A-SH1, RBM8A-SH2 and RMB8A-OE cell lines. (D) Quantification of invasion efficiency.

\section{Discussion}

RBM8A is an RNA binding protein with molecular weight of $26 \mathrm{kDa}$ locating at chromosome 14q21-q23. The gene codes four transcripts and expresses broadly within the cell and shuffles between the nuclear and cytoplasm (14). Compared to other RNA binding motif proteins, the structure and molecular function of RBM8A is poorly understood. The known biological function is that RBM8A could participate in forming the exon-junction binding complex (EJC) (15). RBM8A is the core factor of nonsense-mediated mRNA decay (NMD) and participates in the NMD-mediated mRNA surveillance. NMD keeps the stability of mRNA by degrading the abnormal mRNA products with premature termination codons and reduces the production of harmful shortened proteins (16-18). In 2001, Noensie and Dietz proposed the use of siRNA or drugs to inhibit NMD and in turn to identify mutated genes and treat the tumor (19). Since it has been proposed that the development of malignant tumor is closely related to mRNA stability; it is possible to discover new routes to treat cancer by interfering with the NMD of malignant tumors. As the core factor of NMD, RBM8A may play an essential role during cell malignant transformation.
RBM8A is found highly expressed in malignant tumors such as primary liver cancer, pleural endotheliomas, multiple myeloma and other malignant tumors $(3,11,12,20-23)$. Further analysis revealed that the function of RBM8A could be that the knockdown of RBM8A in lung adenocarcinoma resulted in cell cycle arrest in G2/M phase. RBM8A-Mago complex mediated the location of centrosome which further inhibited the mitosis of tumor cells and then induced apoptosis $(9,24)$. Moreover, as the core factor of EJC complex, loss of RBM8A led to downregulation of the apoptotic genes $\mathrm{Bcl}-\mathrm{xS}$ in Bcl-2 family and Mcl1 and resulted in loss of apoptotic control and further induced the metastasis of hepatocellular carcinoma (HCC) $(2,10)$. Ras/MAPK, JAK/STAT3 and NF-kB signaling pathways are confirmed to be closely related to occurrence, development, invasion and metastasis of liver cancer (25-27). Loss of RBM8A led to changes of all these signaling pathways (23,28-30): loss of RBM8A led to reduction of MAPK protein and inhibited Ras/MAPK signaling pathway which resulted in cell apoptosis; loss of RBM8A resulted in inhibition of JAK/ STAT3 signaling pathway and prohibited bindings between DNA and STAT3; loss of RBM8A also led to decrease of phosphorylation of TNF- $\alpha /$ STAT3 signaling pathway and reduced the effect of NF- $\mathrm{KB}$. As a common factor of these 
A

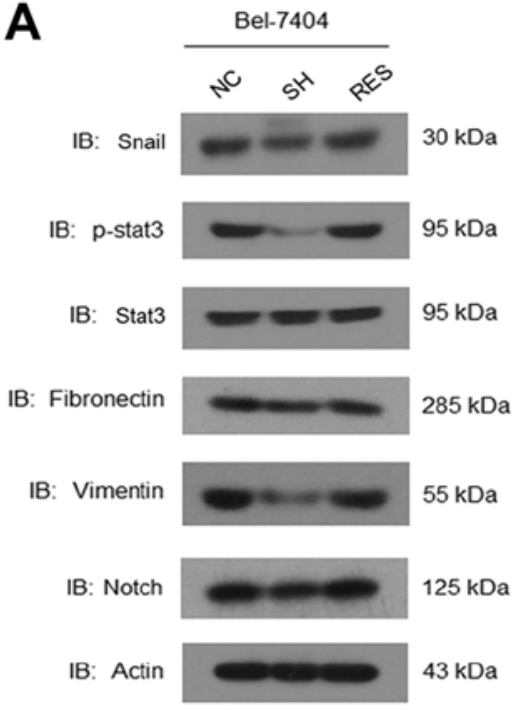

B

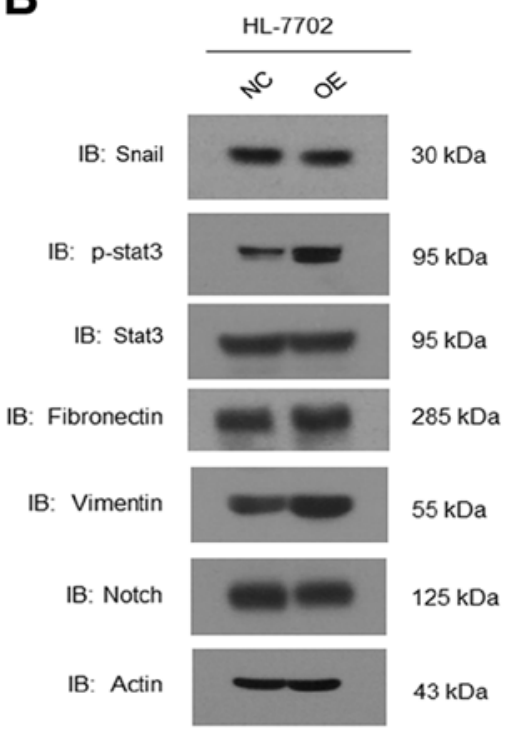

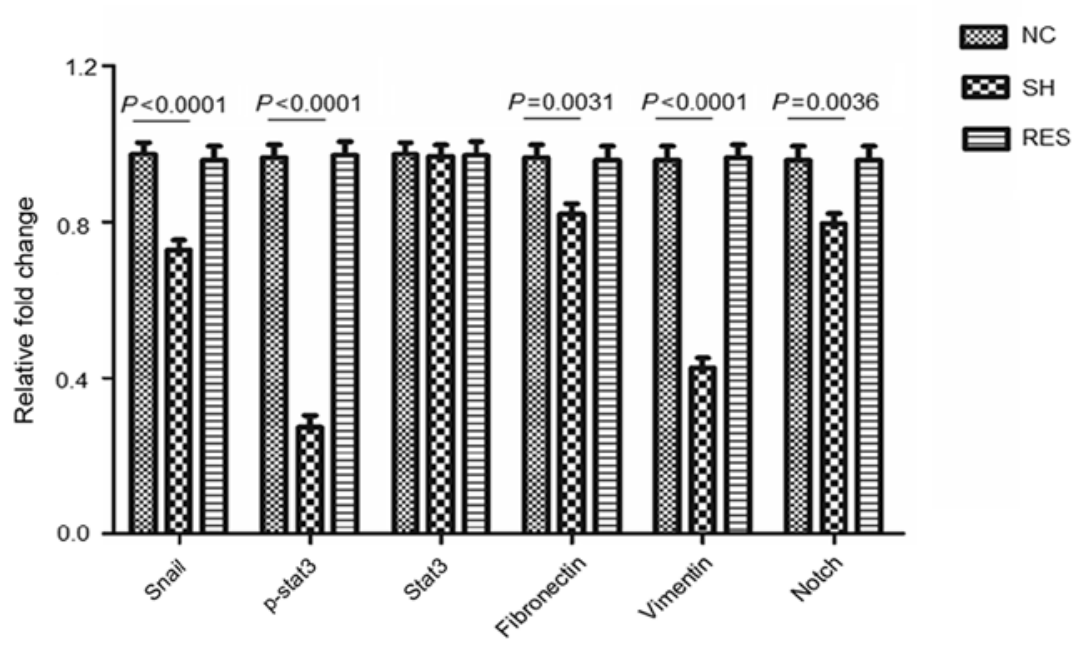
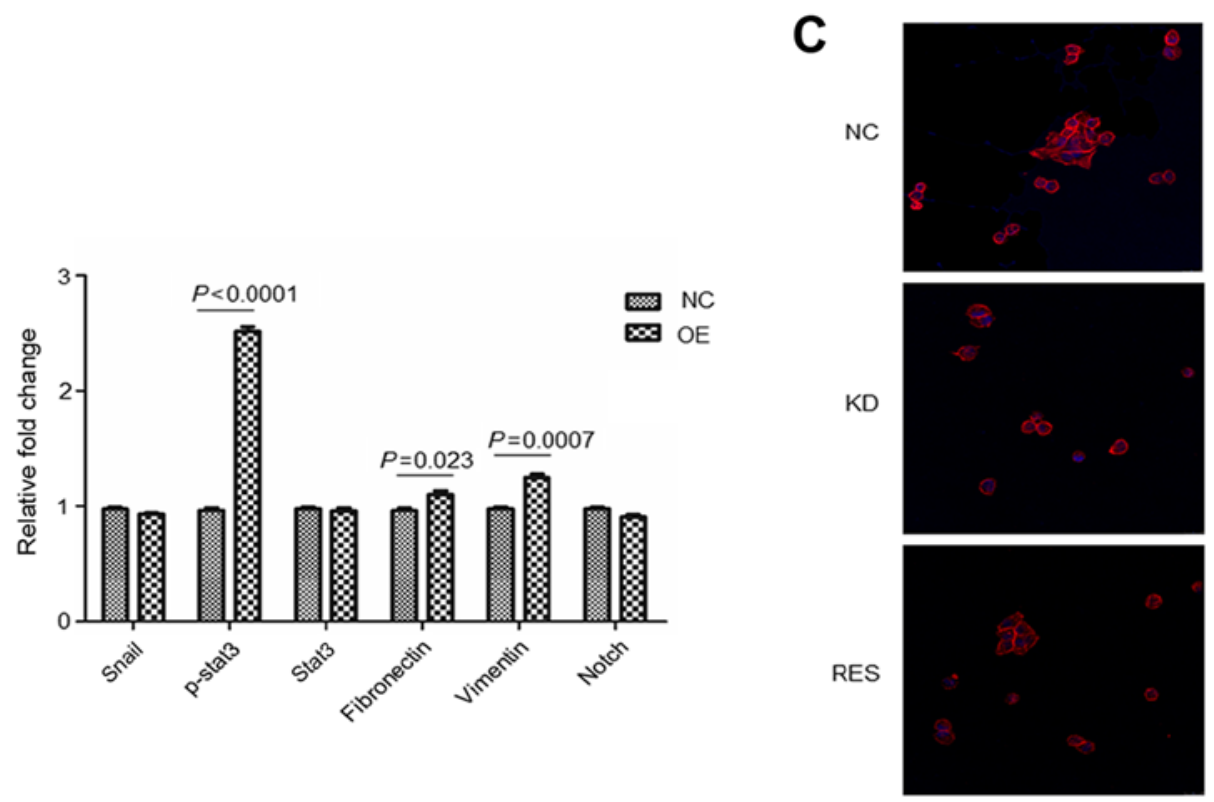

Figure 5. RBM8A regulates HCC cell EMT transition. (A) Expression changes of EMT related genes upon RBM8A knockdown. Quantitative data of western blot results were analyzed. (B) Expression changes of EMT related genes upon RBM8A overexpression. Quantitative data of western blot results were analyzed. (C) Immunofluorescence staining of EMT-related genes.

three signaling pathways, RBM8A protein plays vital roles in inhibiting cell growth and apoptosis. Our results also indicated that RBM8A inhibited apoptosis and loss of RBM8A would clearly prevent invasion and metastasis. Our data showed that EMT signaling pathways were affected upon RBM8A demonstrating that the stability of invasion and metastasis was disrupted due to abnormal EMT process.

Our studies revealed from both in vivo and in vitro level that RBM8A is significantly increased in HCC and could be used as a diagnostic marker for HCC. One of the mechanisms of malignant tumor occurrence is the high mutation frequency of oncogenes. These mutations induce NMD pathway and result in pre-inhibition of oncogene translation. The high expression of RBM8A in HCC tissues verified that NMD pathway is highly active in liver cancer tissues indicating that PTC mutation is higher compared to normal tissues. Furthermore, additional study is required to prove whether micro-metastasis exists and causes differentially expressed RBM8A.

RBM8A expression is associated with HbsAg expression level. Epidemiology results revealed that hepatitis $B$ virus infection is the most critical factor of HCC in China. Almost all the hepatitis B virus-associated liver cancers contain hepatitis B virus integration and the integrated virus promoted the development of liver fibration and liver cell inflammation and in turn promoted the occurrence of liver cancer $(31,32)$. Our result found that significantly high expression of RBM8A at both mRNA and protein level in HbsAg positive group indicating that RBM8A participates in the integration of $\mathrm{Hbs} \mathrm{Ag}$ gene and interacts with hepatitis B virus surface antigen and promotes the occurrence of liver cancer.

Clinical grading and clinical TNM staging are vital for clinical evaluation of liver cancer and provide great significance for prognosis (33). Our results showed that RBM8A 
A

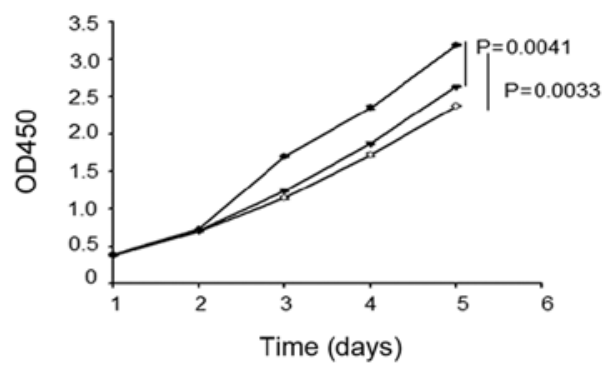

C
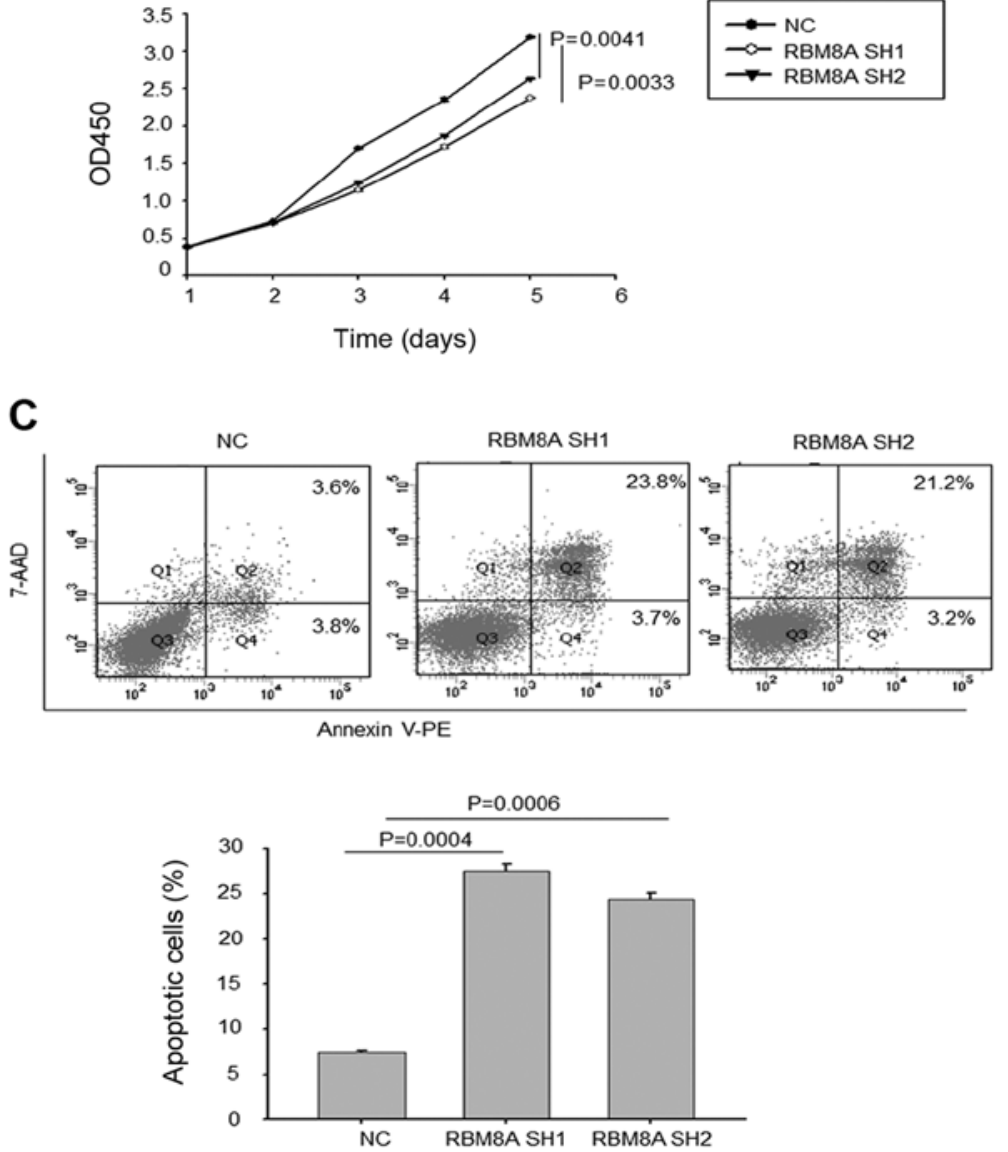

B

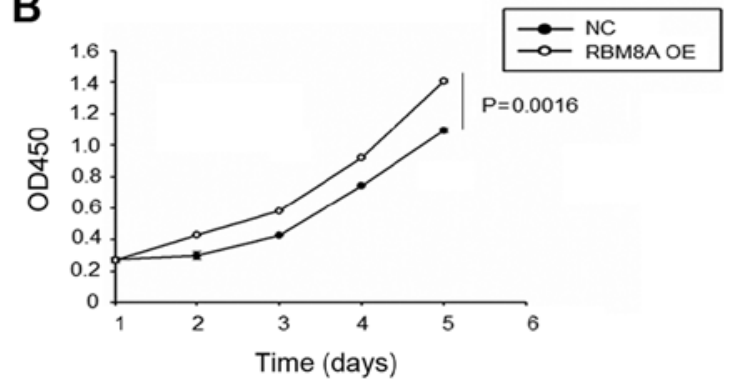

D
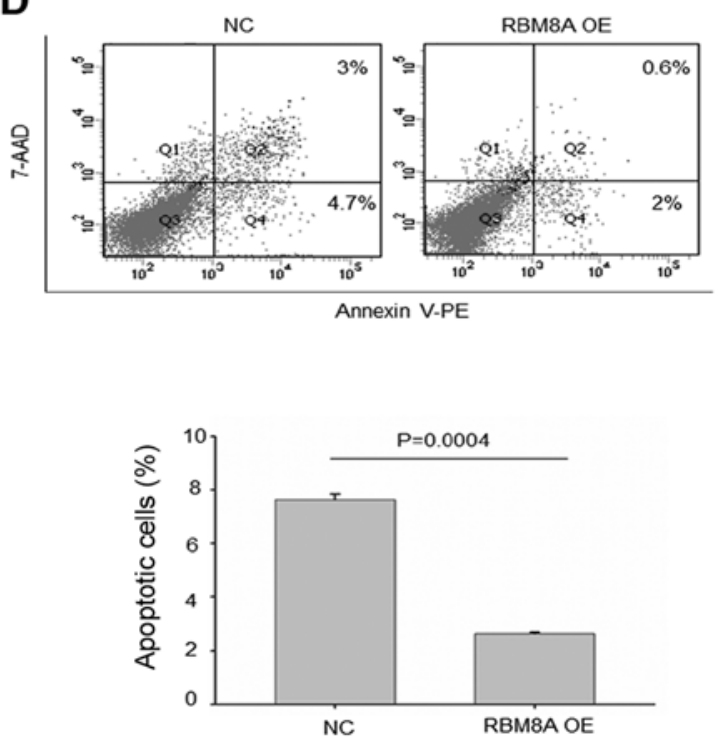

Figure 6. RBM8A plays important function in HCC cell proliferation and survival. (A) HCC population alteration upon RBM8A knockdown (SH1 and SH2). (B) HCC population alteration upon RBM8A overexpression. (C) FACS and quantification of apoptotic cells upon RBM8A knockdown (SH1 and SH2).

(D) FACS and quantification of apoptotic cells upon RBM8A overexpression.

expression is higher in late stage liver cancer than early stage liver cancer. In histology, lower liver cancer pathological grading corresponds to higher RBM8A expression level. Kim et al (21), confirmed that cervical carcinoma patients with lymphatic metastasis possessed higher RBM8A expression than patients without lymphatic metastasis indicating that RBM8A could serve as a molecular marker for liver cancer early diagnosis and metastasis evaluation.

We divided clinical cases into RBM8A high expression level group and RBM8A low expression level group based on IHC results and followed up these cases. Our results showed that median of overall survival are 160 and 229 days, respectively, while median of progression-free survival for RBM8A high expression group and RBM8A low expression group are 90 and 114 days, respectively, indicating that RBM8A could serve as a marker for liver cancer prognosis since RBM8A is closely related to reoccurrence, migration and overall survival.

In summary, our results demonstrated that RBM8A is highly expressed in HCC. RBM8A displayed significant differences in different HBsAg expression, tumor diameter, TNM staging and Edmondson pathological grading which guides pathological and clinical conclusion. Additionally, we found that patients with lower RBM8A expression level had longer OS and PFS. Besides, our results indicate that RBM8A promotes invasion and metastasis via EMT signaling pathway.
Loss of RBM8A significantly induces apoptosis and reduces tumor cell population demonstrating that RBM8A could serve as a potential target for clinical treatment.

\section{Acknowledgements}

This study was supported by Self-Raised Scientific Research Fund of the Ministry of Health of Guangxi Province (no. Z2015605), the Regional Science Fund Project of China Natural Science Foundation (no. 81660498), the China Postdoctoral Science Foundation, the 60th Grant Funding of General Program for the Postdoctoral Funding Program in the Western Region (no. 2016M602919XB).

\section{References}

1. Perz JF, Armstrong GL, Farrington LA, Hutin YJ and Bell BP: The contributions of hepatitis $B$ virus and hepatitis $C$ virus infections to cirrhosis and primary liver cancer worldwide. $\mathrm{J}$ Hepatol 45: 529-538, 2006.

2. Lei X, Li YF, Chen GD, Ou DP, Qiu XX, Zuo CH and Yang LY: Ack1 overexpression promotes metastasis and indicates poor prognosis of hepatocellular carcinoma. Oncotarget 6: 40622-40641, 2015.

3. Salicioni AM, Xi M, Vanderveer LA, Balsara B, Testa JR, Dunbrack RL Jr and Godwin AK: Identification and structural analysis of human RBM8A and RBM8B: Two highly conserved RNA-binding motif proteins that interact with OVCA1, a candidate tumor suppressor. Genomics 69: 54-62, 2000. 
4. Bechara EG, Sebestyén E, Bernardis I, Eyras E and Valcárcel J: RBM5, 6, and 10 differentially regulate NUMB alternative splicing to control cancer cell proliferation. Mol Cell 52: 720-733, 2013.

5. Boman K, Segersten U, Ahlgren G, Eberhard J, Uhlén M, Jirström K and Malmström PU: Decreased expression of RNA-binding motif protein 3 correlates with tumour progression and poor prognosis in urothelial bladder cancer. BMC Urol 13: 17, 2013.

6. Ehlén A, Brennan DJ, Nodin B, O'Connor DP, Eberhard J, Alvarado-Kristensson M, Jeffrey IB, Manjer J, Brändstedt J, Uhlén M, et al: Expression of the RNA-binding protein RBM3 is associated with a favourable prognosis and cisplatin sensitivity in epithelial ovarian cancer. J Transl Med 8: 78, 2010.

7. Fushimi K, Ray P, Kar A, Wang L, Sutherland LC and Wu JY: Up-regulation of the proapoptotic caspase 2 splicing isoform by a candidate tumor suppressor, RBM5. Proc Natl Acad Sci USA 105: 15708-15713, 2008.

8. Jögi A, Brennan DJ, Rydén L, Magnusson K, Fernö M, Stål O, Borgquist S, Uhlen M, Landberg G, Påhlman S, et al: Nuclear expression of the RNA-binding protein RBM3 is associated with an improved clinical outcome in breast cancer. Mod Pathol 22: 1564-1574, 2009.

9. Ishigaki Y, Nakamura Y, Tatsuno T, Hashimoto M, Shimasaki T, Iwabuchi $\mathrm{K}$ and Tomosugi N: Depletion of RNA-binding protein RBM8A (Y14) causes cell cycle deficiency and apoptosis in human cells. Exp Biol Med (Maywood) 238: 889-897, 2013.

10. Michelle L, Cloutier A, Toutant J, Shkreta L, Thibault P, Durand M, Garneau D, Gendron D, Lapointe E, Couture S, et al: Proteins associated with the exon junction complex also control the alternative splicing of apoptotic regulators. Mol Cell Biol 32: 954-967, 2012

11. Sudo H, Tsuji AB, Sugyo A, Kohda M, Sogawa C, Yoshida C, Harada YN, Hino O and Saga T: Knockdown of COPA, identified by loss-of-function screen, induces apoptosis and suppresses tumor growth in mesothelioma mouse model. Genomics 95: 210-216, 2010

12. Zindy PJ, L'Helgoualc'h A, Bonnier D, Le Béchec A, BourdBoitin K, Zhang CX, Musso O, Glaise D, Troadec MB, Loréal O, et al: Upregulation of the tumor suppressor gene menin in hepatocellular carcinomas and its significance in fibrogenesis. Hepatology 44: 1296-1307, 2006.

13. Li Y, Tian B, Yang J, Zhao L, Wu X, Ye SL, Liu YK and Tang ZY: Stepwise metastatic human hepatocellular carcinoma cell model system with multiple metastatic potentials established through consecutive in vivo selection and studies on metastatic characteristics. J Cancer Res Clin Oncol 130: 460-468, 2004

14. Maderazo AB, Belk JP, He F and Jacobson A: Nonsensecontaining mRNAs that accumulate in the absence of a functional nonsense-mediated mRNA decay pathway are destabilized rapidly upon its restitution. Mol Cell Biol 23: 842-851, 2003.

15. Chuang TW, Chang WL, Lee KM and Tarn WY:The RNA-binding protein Y14 inhibits mRNA decapping and modulates processing body formation. Mol Biol Cell 24: 1-13, 2013

16. Gehring NH, Neu-Yilik G, Schell T, Hentze MW and Kulozik AE: Y14 and hUpf $3 b$ form an NMD-activating complex. Mol Cell 11: 939-949, 2003

17. Hilleren P and Parker R: mRNA surveillance in eukaryotes: Kinetic proofreading of proper translation termination as assessed by mRNP domain organization? RNA 5: 711-719, 1999

18. Ishigaki Y, Nakamura Y, Tatsuno T, Ma S and Tomosugi N Phosphorylation status of human RNA-binding protein $8 \mathrm{~A}$ in cells and its inhibitory regulation by Magoh. Exp Biol Med (Maywood) 240: 438-445, 2015.
19. Noensie EN and Dietz HC: A strategy for disease gene identification through nonsense-mediated mRNA decay inhibition. Nat Biotechnol 19: 434-439, 2001.

20. Carrasco DR, Tonon G, Huang Y, Zhang Y, Sinha R, Feng B, Stewart JP, Zhan F, Khatry D, Protopopova M, et al: Highresolution genomic profiles define distinct clinico-pathogenetic subgroups of multiple myeloma patients. Cancer Cell 9: 313-325, 2006.

21. Kim TJ, Choi JJ, Kim WY, Choi CH, Lee JW, Bae DS, Son DS, Kim J, Park BK, Ahn G, et al: Gene expression profiling for the prediction of lymph node metastasis in patients with cervical cancer. Cancer Sci 99: 31-38, 2008.

22. Knuutila S, Aalto Y, Autio K, Björkqvist AM, El-Rifai W, Hemmer S, Huhta T, Kettunen E, Kiuru-Kuhlefelt S, Larramendy ML, et al: DNA copy number losses in human neoplasms. Am J Pathol 155: 683-694, 1999.

23. Togi S, Shiga K, Muromoto R, Kato M, Souma Y, Sekine Y, Kon S, Oritani K and Matsuda T: Y14 positively regulates TNF- $\alpha$-induced NF- $\kappa B$ transcriptional activity via interacting RIP1 and TRADD beyond an exon junction complex protein. J Immunol 191: 1436-1444, 2013.

24. Ishigaki Y, Nakamura Y, Tatsuno T, Hashimoto M, Iwabuchi K and Tomosugi N: RNA-binding protein RBM8A (Y14) and MAGOH localize to centrosome in human A549 cells. Histochem Cell Biol 141: 101-109, 2014.

25. Arsura M and Cavin LG: Nuclear factor-kappaB and liver carcinogenesis. Cancer Lett 229: 157-169, 2005.

26. Calvisi DF, Ladu S, Gorden A, Farina M, Conner EA, Lee JS, Factor VM and Thorgeirsson SS: Ubiquitous activation of Ras and Jak/Stat pathways in human HCC. Gastroenterology 130 : 1117-1128, 2006.

27. Wang C, Cigliano A, Delogu S, Armbruster J, Dombrowski F, Evert M, Chen X and Calvisi DF: Functional crosstalk between AKT/mTOR and Ras/MAPK pathways in hepatocarcinogenesis: Implications for the treatment of human liver cancer. Cell Cycle 12: 1999-2010, 2013

28. Ohbayashi N, Taira N, Kawakami S, Togi S, Sato N, Ikeda O, Kamitani S, Muromoto R, Sekine Y and Matsuda T: An RNA biding protein, Y14 interacts with and modulates STAT3 activation. Biochem Biophys Res Commun 372: 475-479, 2008.

29. Roignant JY and Treisman JE: Exon junction complex subunits are required to splice Drosophila MAP kinase, a large heterochromatic gene. Cell 143: 238-250, 2010.

30. Yang $L$ and Baker NE: Cell cycle withdrawal, progression, and cell survival regulation by EGFR and its effectors in the differentiating Drosophila eye. Dev Cell 4: 359-369, 2003.

31. Brechot C, Kremsdorf D, Soussan P, Pineau P, Dejean A, Paterlini-Brechot $\mathrm{P}$ and Tiollais P: Hepatitis B virus (HBV)related hepatocellular carcinoma (HCC): Molecular mechanisms and novel paradigms. Pathol Biol (Paris) 58: 278-287, 2010.

32. Xu C, Zhou W, Wang Y and Qiao L: Hepatitis B virus-induced hepatocellular carcinoma. Cancer Lett 345: 216-222, 2014.

33. Yan T, Zhao JJ, Bi XY, Zhao H, Huang Z, Li ZY, Zhou JG, Li Y, Li C, Cai JQ, et al: Prognosis of hepatocellular carcinoma: A study of 832 cases. Zhonghua Zhong Liu Za Zhi 35: 54-58, 2013 (In Chinese). 